\title{
Ensemble Averaged Conservation Equations For Multiphase, Multi-Component, And Multi-Material FLows
}

R. A. Berry, Ph.D.

August 2003

Idaho National Engineering and Environmental Laboratory Bechtel BWXT Idaho, LLC 


\title{
Preface
}

At the INEEL researchers and engineers routinely encounter multiphase, multi-component, and/or multi-material flows. Some examples include:

\author{
Reactor coolant flows \\ Molten corium flows \\ Dynamic compaction of metal powders \\ Spray forming and thermal plasma spraying \\ Plasma quench reactor \\ Subsurface flows, particularly in the vadose zone \\ Internal flows within fuel cells \\ Black liquor atomization and combustion \\ Wheat-chaff classification in combine harvesters \\ Generation IV pebble bed, high temperature gas reactor
}

The complexity of these flows dictates that they be examined in an averaged sense. Typically one would begin with known (or at least postulated) microscopic flow relations that hold on the "small" scale. These include continuum level conservation of mass, balance of species mass and momentum, conservation of energy, and a statement of the second law of thermodynamics often in the form of an entropy inequality (such as the Clausius-Duhem inequality). The averaged or macroscopic conservation equations and entropy inequalities are then obtained from the microscopic equations through suitable averaging procedures. At this stage a stronger form of the second law may also be postulated for the mixture of phases or materials. To render the evolutionary material flow balance system unique, constitutive equations and phase or material interaction relations are introduced from experimental observation, or by postulation, through strict enforcement of the constraints or restrictions resulting from the averaged entropy inequalities. These averaged equations form the governing equation system for the dynamic evolution of these mixture flows.

Most commonly, the averaging technique utilized is either volume or time averaging or a combination of the two. The flow restrictions required for volume and time averaging to be valid can be severe, and violations of these restrictions are often found. A more general, less restrictive (and far less commonly used) type of averaging known as ensemble averaging can also be used to produce the governing equation systems. In fact volume and time averaging can be viewed as special cases of ensemble averaging. Ensemble averaging is beginning to gain some notice, for example the general-purpose multi-material flow simulation code CFDLib under continuing developed at the Los Alamos National Laboratory [Kashiwa and Rauenzahn 1994] is based on an ensemble averaged formulation.

The purpose of this short note is to give an introduction to the ensemble averaging methodology and to show how ensemble averaged balance equations and entropy inequality can be obtained from the microscopic balances. 


\section{Ensemble Averaged Conservation Equations For Multiphase, Multi-Component, And Multi-Material Flows}

\section{Introduction}

Many important "fluid" flows involve a combination of two or more materials having different properties. The multiple phases or components often exhibit relative motion among the phases or material classes. The microscopic motions of the individual constituents are complex and the solution to the micro-level evolutionary equations is difficult. Characteristic of such flows of multi-component materials is an uncertainty in the exact locations of the particular constituents at any particular time. For most practical purposes, it is not possible to exactly predict or measure the evolution of the details of such systems, nor is it even necessary or desirable. Instead, we are usually interested in more gross features of the motion, or the "average" behavior of the system. Here we present descriptive equations that will predict the evolution of this averaged behavior. Due to the complexities of interfaces and resultant discontinuities in fluid properties, as well as from physical scaling issues, it is essential to work with averaged quantities and parameters. We begin by tightening up, or more rigorously defining, our concept of an average. There are several types of averaging. The published literature predominantly contains two types of averaging: volume averaging [Whitaker 1999, Dobran 1991] and time averaging [Ishii 1975]. Occasionally combinations of the two are used. However, we utilize a more general approach by adopting what is known as ensemble averaging.

When the physical system has a large amount of variability, a natural interpretation of the meaning of predictions is in terms of expected values and variances. If there are many different events, or realizations, possible, then the expected value is naturally an "average" over all of these events, or the ensemble of realizations. The ensemble then is the set of all experiments with the same boundary- and initial-conditions, with some properties that we would like to associate with the mean and distribution of the components and their velocities. A realization of the flow is a possible motion that could have happened. Implicit in this concept is the intuitive idea of a "more likely" and a "less likely" realization in the ensemble. Therefore, as we shall see shortly each ensemble of realizations, corresponding to a given physical situation, has a probability measure on subsets of realizations. The ensemble average is the generalization of the elementary idea of adding the values of the variable for each realization, and dividing by the number of observations. The ensemble average then allows the interpretation of phenomena in terms of repeatability of multi-component flows.

One of the nice features of ensemble averaging, as opposed to volume averaging, is that ensemble averaging does not require that a control volume contain a large quantity of a particular component in any given realization. Consider the following example, taken directly from Drew and Lahey (1993), where the average of a particle-fluid mixture is of interest. Gas turbines are 
eroded by particulate matter suspended in the gas stream passing through the inlet and impacting on the various parts of the machine, e.g. the turbine blades. The trajectories of individual particles moving through the gas turbine are very complicated, depending on where and when the particles enter the inlet of the device. Such predictions are, fortunately, seldom required. A prediction, however, that is of interest to the designer is the average, or expected values, of the particle flux (or the concentration and velocities of particles) near parts in the device that are susceptible to erosion. Since the local concentration of particles is proportional to the probability that particles will be at the various points in the device at various times, and the particle velocity field will be the mean velocity that the particles will have if they are at that position in the device, the design engineer will be able to use this information to assess the places where erosion due to particle impact may occur. Notice it may be that there are no times for which there will be many particles in some representative control volume (or representative elementary volume, REV). So, volume averaging, which depends on the concept of having many representative particles in the averaging volume at any instant, will fail. The appropriateness of ensemble averaging is obvious. Here the ensemble is the set of motions of a single particle through the device, given that it started at a random point at the inlet at a random time during the transient flow through the device. Clearly the solution for the average concentration and average velocity gives little information about the behavior of a single particle in the device; however, the information is very appropriate for assessing the probability of damage to the device. Similar examples could be given where time averaging will fail, but where ensemble averaging is again appropriate.

The ensemble average is the more fundamentally based than either time or volume averaging. In fact, both time and volume averaging can be viewed as approximations to the ensemble average, which can be justified, respectively, for steady or homogeneous flow [Drew and Passman 1999].

\section{Ensemble Averaging}

A general method is presented here, based on the ensemble averaging concept, for developing averaged conservation equations for multiple materials, any one of which may be at point $\vec{x}$, at a given instant $t$. With this procedure, the most likely state at a point (the expected value) will be determined simultaneously with which material is most likely to be found at that point. Imagine running an experiment many times and collecting data about the state of the flow at each point $\vec{x}$ and time $t$. This information could include which material or phase is present, material density, velocity, pressure, temperature, concentration, etc. From this information, one can compute the ensemble average. The ensemble average of a generic property $Q_{0}$ of a fluid or material in a process is an average over the realizations

$$
\left\langle Q_{0}\right\rangle(\vec{x}, t)=\frac{1}{N_{R}} \sum_{r=1}^{N_{R}} Q_{0, r}(\vec{x}, t),
$$

where $N_{R}$ is the number of times the process or experiment is repeated, and is a large number. Now imagine that many of the realizations are near duplicates, i.e. they are essentially the same 
state, with $N$ occurrences. We can then rewrite the sum over the realizations as a sum over the number of states $N_{\Gamma}$

$$
\begin{aligned}
\left\langle Q_{0}\right\rangle(\vec{x}, t) & =\frac{1}{N_{R}} \sum_{\Gamma=1}^{N_{\Gamma}} N(\vec{x}, t, \Gamma) Q_{0}(\Gamma) \\
& =\sum_{\Gamma=1}^{N_{\Gamma}} \frac{N(\vec{x}, t, \Gamma)}{N_{R}} Q_{0}(\Gamma) \\
& =\int_{\text {all } \Gamma} Q_{0}(\Gamma) f(\vec{x}, t, \Gamma) d \Gamma,
\end{aligned}
$$

where $f(\vec{x}, t, \Gamma)=\frac{N(\vec{x}, t, \Gamma)}{N_{R}}$ is the probability of the state $\Gamma$ in the ensemble. Note that in the limit of an infinite number of repetitions of the experiment, with a sum over all of the states, we have replaced the summation with an integral form in the definition of the ensemble average. More correctly, because $\int_{\text {all } \Gamma} f(\vec{x}, t, \Gamma) d \Gamma=1.0$, we refer to $f(\vec{x}, t, \Gamma)$ as the probability density.

The state is the full thermodynamic description of the matter at a point $\vec{x}$ and time t. For example,

$$
\Gamma=\left\{\begin{array}{l}
\rho_{0}, \vec{u}_{0}, h_{0}, p_{0}, \tau_{\sim}, \\
\rho_{0}^{1}, \vec{u}_{0}^{1}, h_{0}^{1}, \rho_{0}^{2}, \vec{u}_{0}^{2}, h_{0}^{2}, \cdots, \\
X_{1}, X_{2}, \cdots
\end{array}\right\}
$$

where:

$X_{k}(\vec{x}, t) \quad$ phase or material indicator function $:=1$ if material $k$ is present

$$
=0 \text { otherwise }
$$

$\rho_{0} \quad$ phase or material density

$\vec{u}_{0} \quad$ phase or material velocity

$h_{0} \quad$ phase or material specific enthalpy

$p_{0} \quad$ pressure

$\tau_{0} \quad$ deviatoric stress

$\rho_{0}^{s} \quad$ species partial density

$\vec{u}_{0}^{s} \quad$ species velocity

$h_{0}^{s} \quad$ species partial enthalpy,

with 


$$
\begin{aligned}
& \rho_{0}=\sum_{\text {species }} \rho_{0}^{s} \\
& \rho_{0} \vec{u}_{0}=\sum_{\text {species }} \rho_{0}^{s} \vec{u}_{0}^{s} \\
& \rho_{0} h_{0}=\sum_{\text {species }} \rho_{0}^{s} h_{0}^{s} .
\end{aligned}
$$

Other properties may also appear in the above thermodynamic state such as the phase or material temperature $\theta_{0}$, the phase or material specific internal energy $e_{0}$, and the phase or material specific entropy $s_{0}$.

In a typical multiphase flow, the ensemble averages of interest may include

$$
\begin{aligned}
& \text { Material } k \text { volume fraction : } \quad \alpha_{k} \equiv\left\langle X_{k}\right\rangle \\
& \text { Material } k \text { bulk average density : } \quad \hat{\rho}_{k} \equiv\left\langle X_{k} \rho_{0}\right\rangle \\
& \text { Material } k \text { intrinsic average density : } \quad \rho_{k} \equiv \frac{\left\langle X_{k} \rho_{0}\right\rangle}{\alpha_{k}} \\
& \text { Species s in material } k \text { bulk average density : } \quad \hat{\rho}_{k}^{s} \equiv\left\langle X_{k} \rho_{0}^{s}\right\rangle \\
& \text { Species } s \text { in material } k \text { intrinsic average density : } \quad \rho_{k}^{s} \equiv \frac{\left\langle X_{k} \rho_{0}^{s}\right\rangle}{\alpha_{k}} \\
& \text { Material } k \text { velocity : } \quad \vec{u}_{k} \equiv \frac{\left\langle X_{k} \rho_{0} \vec{u}_{0}\right\rangle}{\hat{\rho}_{k}}=\frac{\left\langle X_{k} \rho_{0} \vec{u}_{0}\right\rangle}{\alpha_{k} \rho_{k}} \\
& \text { Material } k \text { total energy : } \quad E_{k} \equiv \frac{\left\langle X_{k} \rho_{0} E_{0}\right\rangle}{\hat{\rho}_{k}}=\frac{\left\langle X_{k} \rho_{0} E_{0}\right\rangle}{\alpha_{k} \rho_{k}} \\
& \text { Material k entropy: } \quad s_{k} \equiv \frac{\left\langle X_{k} \rho_{0} s_{0}\right\rangle}{\hat{\rho}_{k}}=\frac{\left\langle X_{k} \rho_{0} s_{0}\right\rangle}{\alpha_{k} \rho_{k}} \\
& \text { Mean mixture stress : } \quad \underset{\sim}{T} \equiv\langle\underset{\sim}{T}\rangle \\
& \text { Mean } k \text {-material stress : } \quad T_{\sim k} \equiv \frac{\left\langle X_{k} T_{\sim}\right\rangle}{\alpha_{k}} \\
& \text { Pressure (single pressure model) : } \quad p \equiv\left\langle p_{0}\right\rangle \\
& \text { Pressure in } k \text {-material : } \quad p_{k} \equiv \frac{\left\langle X_{k} p_{0}\right\rangle}{\alpha_{k}} \text {. }
\end{aligned}
$$

From a physical viewpoint, the bulk average density of a phase represents a summation of all of the density values that occurred for that phase, divided by the total number of experiments run. The bulk average density corresponds intuitively to the idea of the mass of phase $k$ per unit volume of mixture, or the observed material density. On the other hand, the intrinsic average density physically corresponds to a summation of all of the density values that occurred for that phase, dividing by the number of times in which that phase occurred in the experiments. The 
intrinsic average density corresponds intuitively to the idea of the mass of phase $k$ per unit volume of phase $k$, or the true material density. Some researchers prefer to work with bulk average densities [e.g. Kashiwa and Rauenzahn 1994] while others prefer working with intrinsic densities [e.g. Drew and Passman 1999]. This is mostly an issue of convenience, since one can easily be converted to the other. Here we will use intrinsic averages. Henceforth, when we say average, we shall mean intrinsic average unless indicated otherwise.

For a reasonably broad range of conditions (with common substances), the exact balance equations, valid inside each material, are

$$
\begin{array}{cc}
\dot{\rho}_{0}=-\rho_{0} \nabla \cdot \vec{u}_{0} & \text { Material mass conservation } \\
\dot{\rho}_{0}^{s}=-\rho_{0}^{s} \nabla \cdot \vec{u}_{0}-\nabla \cdot \rho_{0}^{s}\left(\vec{u}_{0}^{s}-\vec{u}_{0}\right)+\dot{r}_{0}^{s} & \text { Species mass conservation } \\
\rho_{0} \dot{\vec{u}}_{0}=\nabla \cdot{\underset{\sim}{T}}_{0}+\rho_{0} \vec{g} & \text { Material momentum balance } \\
\rho_{0} \dot{E}_{0}=\nabla \cdot\left({\underset{\sim}{T} 0}_{T_{0}} \cdot \vec{u}_{0}+\nabla \cdot \vec{q}_{0}+\rho_{0} \vec{g} \cdot \vec{u}_{0}+\rho_{0} \varepsilon_{0}\right. & \text { Material energy conservation } \\
\rho_{0} \dot{s}_{0} \geq \frac{\rho_{0} \varepsilon_{0}}{\theta_{0}}-\nabla \cdot\left(\frac{\vec{q}_{0}}{\theta_{0}}\right) &
\end{array}
$$

For these microscopic balance laws the material derivative has been used, which is defined as

$$
\dot{Q}_{0} \equiv \frac{\partial Q_{0}}{\partial t}+\vec{u}_{0} \cdot \nabla Q_{0} \quad \text { Material derivative }
$$

Let us assume that the total variation of $f$ in the phase space $(\vec{x}, t, \Gamma)$ is [Kashiwa and Rauenzahn 1994]

$$
\frac{\partial f}{\partial t}+\vec{u}_{0} \cdot \nabla f+\dot{\Gamma} \cdot \frac{\partial f}{\partial \Gamma}=\frac{d f}{d t}=0
$$

where we are assuming that as we follow a material point through phase space its probability of occurrence remains constant. Various moments of this equation can be formed, first by multiplying equation (10) by $Q_{0}$, and then averaging this result. It can be shown [Kashiwa and Rauenzahn 1994, here corrected] that the resulting equation is

$$
\frac{\partial}{\partial t}\left\langle Q_{0}\right\rangle+\nabla \cdot\left\langle Q_{0} \vec{u}_{0}\right\rangle=\left\langle\dot{Q}_{0}+Q_{0} \nabla \cdot \vec{u}_{0}\right\rangle
$$

This result is called the moment evolution equation and the details of its derivation are given in the Appendix. The averaged conservation equations are obtained by letting our generic $Q_{0}$ be 
replaced by various "meaningful" functions and then by performing judicious manipulations on the equations to bring about physically useful forms of the equation.

\section{Mass Conservation}

By letting $Q_{0}=X_{k} \rho_{0}$ in equation (11) we get

$$
\begin{aligned}
\frac{\partial\left\langle X_{k} \rho_{0}\right\rangle}{\partial t}+\nabla \cdot\left\langle X_{k} \rho_{0} \vec{u}_{0}\right\rangle & =\left\langle\dot{X}_{k} \rho_{0}+X_{k} \dot{\rho}_{0}+X_{k} \rho_{0} \nabla \cdot \vec{u}_{0}\right\rangle \\
& =\left\langle\dot{X}_{k} \rho_{0}+X_{k}\left(\dot{\rho}_{0}+\rho_{0} \nabla \cdot \vec{u}_{0}\right)\right\rangle
\end{aligned}
$$

Introducing the pure material (microscopic) mass conservation equation and the definition of average results in

$$
\frac{\partial \alpha_{k} \rho_{k}}{\partial t}+\nabla \cdot \alpha_{k} \rho_{k} \vec{u}_{k}=\left\langle\dot{X}_{k} \rho_{0}\right\rangle
$$

Since we are taking time- and spatial-derivatives of functions that are not smooth, this averaged mass conservation equation is to be interpreted in the sense of distributions, or generalized functions [Gelfand and Shilov 1964]. Let us examine the right hand side of this equation in more detail. From the definition of a material derivative we know that

$$
\dot{X}_{k}=\frac{\partial X_{k}}{\partial t}+\vec{u}_{0} \cdot \nabla X_{k}
$$

in a generalized function sense. On the other hand, letting $\vec{u}_{i n t}$ denote the velocity of an interface of phase or material $\mathrm{k}$, the material derivative of $X_{k}$ following the interface velocity vanishes

$$
\frac{\partial X_{k}}{\partial t}+\vec{u}_{i n t} \cdot \nabla X_{k}=0
$$

This result can be easily seen by first considering points not on the interface where either $X_{k}=0$ or $X_{k}=1$ and the partial derivatives both vanish, and thus the left side of this equation vanishes identically. For points on the interface, which also move with the interface velocity, the function $X_{k}$ is a jump that remains constant so their material derivatives following the interface vanish.

Therefore we can write 


$$
\begin{aligned}
\left\langle\dot{X}_{k} \rho_{0}\right\rangle & =\left\langle\rho_{0}[\left(\frac{\partial X_{k}}{\partial t}+\vec{u}_{0} \cdot \nabla X_{k}\right)-\underbrace{\left(\frac{\partial X_{k}}{\partial t}+\vec{u}_{i n t} \cdot \nabla X_{k}\right)}_{=0}]\right\rangle \\
& =\left\langle\rho_{0}\left(\vec{u}_{0}-\vec{u}_{i n t}\right) \cdot \nabla X_{k}\right\rangle,
\end{aligned}
$$

and the averaged mass conservation equation becomes

$$
\begin{aligned}
\frac{\partial \alpha_{k} \rho_{k}}{\partial t}+\nabla \cdot \alpha_{k} \rho_{k} \vec{u}_{k} & =\left\langle\rho_{0}\left(\vec{u}_{0}-\vec{u}_{i n t}\right) \cdot \nabla X_{k}\right\rangle \\
& \equiv \Omega_{k}^{\text {mass }} .
\end{aligned}
$$

We note that $\nabla X_{k}$ has the sifting property of the Dirac delta function(al). Thus the only contributors are the material interfaces. $\nabla X_{k}$ is aligned with the surface unit normal vector pointing to phase $k$ [Drew 1983, Kataoka and Serizawa 1988]

$$
\nabla X_{k}=\vec{n}_{k} \delta\left(\vec{x}-\vec{x}_{i n t}, t\right)
$$

Thus the $\Omega_{k}^{\text {mass }}$ represents the flux of mass to phase $k$ from the other phases via the interface, usually just referred to as phase change. With no storage of mass at an interface the mass conservation further requires

$$
\sum_{k=1}^{\text {no.of phases }} \Omega_{k}^{\text {mass }}=0
$$

At this point, it is convenient to introduce for later use, the concept of interfacial area density of component $k$. Defined as

$$
A_{k}=-\left\langle\hat{n}_{k} \cdot \nabla X_{k}\right\rangle
$$

where $\hat{n}_{k}$ is the unit external normal to component $k$, it is the expected value of the ratio of the interfacial area (in a small volume) to the (small) volume, in the limit as that volume approaches zero. 


\section{Generic Conservation Equation}

To more expeditiously derive the other conservation equations, let us first derive the averaged balance equation resulting from a generic, microscopic balance equation. Consider the generic, microscopic balance equation

$$
\frac{\partial \rho_{0} \psi_{0}}{\partial t}+\nabla \cdot \rho_{0} \psi_{0} \vec{u}_{0}=\nabla \cdot J_{0}+\rho_{0} g_{0}
$$

or

$$
\frac{d\left(\rho_{0} \psi_{0}\right)}{d t}+\left(\rho_{0} \psi_{0}\right) \nabla \cdot \vec{u}_{0}=\rho_{0} \dot{\psi}_{0}=\nabla \cdot J_{0}+\rho_{0} g_{0}
$$

Equation (16) and (17) hold at each point where sufficient smoothness occurs for the derivatives to be taken, as does its generic jump condition

$$
\llbracket \rho_{0} \psi_{0}\left(\vec{u}_{0}-\vec{u}_{i n t}\right)+J_{0} \rrbracket \cdot \hat{n}=m
$$

where $\psi_{0}$ is the conserved quantity, $J_{0}$ is a molecular or diffusive flux, $g_{0}$ is a source density, and $m$ is the interfacial source of $\psi_{0}$. The symbol $\llbracket \cdot \rrbracket$ here denotes the jump in the enclosed quantity across an interface. Obviously, these quantities must be added to our state space, e.g.

$$
\Gamma=\left\{\begin{array}{l}
\rho_{0}, \vec{u}_{0}, \psi_{0}, J_{0}, \cdots \\
X_{1}, X_{2}, \cdots
\end{array}\right\} .
$$

Let us also define averages of these quantities as

$$
\begin{gathered}
\psi_{k} \equiv \frac{\left\langle X_{k} \rho_{0} \psi_{0}\right\rangle}{\alpha_{k} \rho_{k}} \\
J_{k} \equiv \frac{\left\langle X_{k} J_{0}\right\rangle}{\alpha_{k}} \\
g_{k} \equiv \frac{\left\langle X_{k} \rho_{0} g_{0}\right\rangle}{\alpha_{k} \rho_{k}} .
\end{gathered}
$$

By letting $Q_{0}=X_{k} \rho_{0} \psi_{0}$ in equation (11) we get 


$$
\begin{aligned}
\frac{\partial\left\langle X_{k} \rho_{0} \psi_{0}\right\rangle}{\partial t}+\nabla \cdot\left\langle X_{k} \rho_{0} \psi_{0} \vec{u}_{0}\right\rangle & =\left\langle\frac{d\left(X_{k} \rho_{0} \psi_{0}\right)}{d t}+X_{k} \rho_{0} \psi_{0} \nabla \cdot \vec{u}_{0}\right\rangle \\
& =\left\langle\dot{X}_{k} \rho_{0} \psi_{0}+X_{k} \frac{d\left(\rho_{0} \psi_{0}\right)}{d t}+X_{k} \rho_{0} \psi_{0} \nabla \cdot \vec{u}_{0}\right\rangle \\
& =\left\langle\dot{X}_{k} \rho_{0} \psi_{0}\right\rangle+\left\langle X_{k}\left(\frac{d\left(\rho_{0} \psi_{0}\right)}{d t}+\rho_{0} \psi_{0} \nabla \cdot \vec{u}_{0}\right)\right\rangle \\
& =\left\langle\dot{X}_{k} \rho_{0} \psi_{0}+X_{k} \nabla \cdot J_{0}+X_{k} \rho_{0} g_{0}\right\rangle \\
& =\left\langle\nabla \cdot X_{k} J_{0}\right\rangle-\left\langle J_{0} \cdot \nabla X_{k}\right\rangle+\left\langle\dot{X}_{k} \rho_{0} \psi_{0}\right\rangle+\left\langle X_{k} \rho_{0} g_{0}\right\rangle \\
& =\left\langle\nabla \cdot X_{k} J_{0}\right\rangle-\left\langle J_{0} \cdot \nabla X_{k}\right\rangle+\left\langle\rho_{0} \psi_{0}\left(\vec{u}_{0}-\vec{u}_{i n t}\right) \cdot \nabla X_{k}\right\rangle+\left\langle X_{k} \rho_{0} g_{0}\right\rangle \\
& =\nabla \cdot\left\langle X_{k} J_{0}\right\rangle+\left\langle X_{k} \rho_{0} g_{0}\right\rangle+\left\langle\left[\rho_{0} \psi_{0}\left(\vec{u}_{0}-\vec{u}_{i n t}\right)-J_{0}\right] \cdot \nabla X_{k}\right\rangle .
\end{aligned}
$$

Introducing the fluctuating velocity

$$
\vec{u}_{k}^{\prime}=\vec{u}_{0}-\vec{u}_{k}
$$

into this expression finally results in

$$
\begin{aligned}
\frac{\partial \alpha_{k} \rho_{k} \psi_{k}}{\partial t}+\nabla \cdot \alpha_{k} \rho_{k} \psi_{k} \vec{u}_{k} & =\nabla \cdot \alpha_{k} J_{k}-\nabla \cdot\left\langle X_{k} \rho_{0} \psi_{0} \vec{u}_{k}^{\prime}\right\rangle+\alpha_{k} \rho_{k} g_{k}+\left\langle\left[\rho_{0} \psi_{0}\left(\vec{u}_{0}-\vec{u}_{i n t}\right)-J_{0}\right] \cdot \nabla X_{k}\right\rangle \\
& =\nabla \cdot \alpha_{k} J_{k}-\nabla \cdot\left\langle X_{k} \rho_{0} \psi_{0} \vec{u}_{k}^{\prime}\right\rangle+\alpha_{k} \rho_{k} g_{k}+\left\langle\rho_{0} \psi_{0}\left(\vec{u}_{0}-\vec{u}_{i n t}\right) \cdot \nabla X_{k}\right\rangle-\left\langle J_{0} \cdot \nabla X_{k}\right\rangle \\
& =\nabla \cdot \alpha_{k} J_{k}+\nabla \cdot \alpha_{k} J_{k}^{\text {Fluct }}+\alpha_{k} \rho_{k} g_{k}+\Omega_{k}^{\text {mass }} \psi_{k}^{\text {int }}+\Omega_{k}^{\psi},
\end{aligned}
$$

where $J_{k}^{\text {Fluct }}=-\frac{\left\langle X_{k} \rho_{0} \psi_{0} \vec{u}_{k}^{\prime}\right\rangle}{\alpha_{k}}$ is the flux of $\psi$ due to fluctuations in the phase $k$ velocity, $\psi_{k}^{\text {int }}$ is the effective value of $\psi$ that is transferred to phase $k$ from the other phases due to mass transfer, or phase change, and $\Omega_{k}^{\psi}$ is a flux of $\psi$ to phase $k$ not due to bulk mass transfer from the other phases. This is our generic, averaged balance equation. To obtain balance at the interface, our generic jump balance equation requires the constraint

$$
\sum_{k=1}^{\text {no. of phases }} \Omega_{k}^{\text {mass }} \psi_{k}^{\text {int }}+\Omega_{k}^{\psi}=M
$$

where $M=\langle m\rangle$ is the expected net effect of all the interfacial $\psi$-source terms. 


\section{Species Mass Conservation}

The microscopic species mass balance equation can be written as

$$
\frac{\partial \rho_{0}^{s}}{\partial t}+\nabla \cdot \rho_{0}^{s} \vec{u}_{0}^{s}=\dot{r}^{s}
$$

where $\rho_{0}^{s}$ is the species partial density, $\vec{u}_{0}^{s}$ is the species bulk velocity, and $\dot{r}^{s}$ is the generation or source of the species due to chemical reactions. The species mass balance equation is not usually written this way because we usually don't know much about individual species velocities. Instead, it is usually cast as

$$
\frac{\partial \rho_{0}^{s}}{\partial t}+\nabla \cdot \rho_{0}^{s} \vec{u}_{0}=\nabla \cdot \rho_{0}^{s}\left(\vec{u}_{0}-\vec{u}_{0}^{s}\right)+\dot{r}^{s}
$$

because we have (to a certain extent) acquired empirical knowledge of the behavior of the first term on the right hand side of this equation, as we shall see shortly. Let us now recast this equation as

$$
\frac{\partial}{\partial t}\left(\rho_{0} \frac{\rho_{0}^{s}}{\rho_{0}}\right)+\nabla \cdot\left(\rho_{0} \frac{\rho_{0}^{s}}{\rho_{0}} \vec{u}_{0}\right)=\nabla \cdot\left[\rho_{0} \frac{\rho_{0}^{s}}{\rho_{0}}\left(\vec{u}_{0}-\vec{u}_{0}^{s}\right)\right]+\rho_{0} \frac{\dot{r}^{s}}{\rho_{0}}
$$

which is in the form of our generic, averaged balance equation (20) with the assignments of

$$
\psi_{0}=\frac{\rho_{0}^{s}}{\rho_{0}} \quad J_{0}=\rho_{0} \frac{\rho_{0}^{s}}{\rho_{0}}\left(\vec{u}_{0}-\vec{u}_{0}^{s}\right) \quad g_{0}=\frac{\dot{r}^{s}}{\rho_{0}} .
$$

Thus the averaged species mass balance equation is

$$
\frac{\partial}{\partial t}\left\langle X_{k} \rho_{0}^{s}\right\rangle+\nabla \cdot\left\langle X_{k} \rho_{0}^{s} \vec{u}_{0}\right\rangle=\nabla \cdot\left\langle X_{k} \rho_{0}^{s}\left(\vec{u}_{0}-\vec{u}_{0}^{s}\right)\right\rangle+\left\langle X_{k} \dot{r}^{s}\right\rangle+\left\langle\left[\rho_{0}^{s}\left(\vec{u}_{0}-\vec{u}_{i n t}\right)-\rho_{0}^{s}\left(\vec{u}_{0}-\vec{u}_{0}^{s}\right)\right] \cdot \nabla X_{k}\right\rangle \cdot
$$

Again introducing the fluctuating velocity and the definitions of averaged quantities, our final form of the averaged species mass balance equation is

$$
\begin{aligned}
\frac{\partial \alpha_{k} \rho_{k}^{s}}{\partial t}+\nabla \cdot \alpha_{k} \rho_{k}^{s} \vec{u}_{k} & =\nabla \cdot\left\langle X_{k} \rho_{0}^{s}\left(\vec{u}_{0}-\vec{u}_{0}^{s}\right)\right\rangle & & \text { relative species flux } \\
& -\nabla \cdot\left\langle X_{k} \rho_{0}^{s} \vec{u}_{k}^{\prime}\right\rangle & & \text { fluctuational diffusion } \\
& +\left\langle\rho_{0}^{s}\left(\vec{u}_{0}-\vec{u}_{i n t}\right) \cdot \nabla X_{k}\right\rangle & & \text { phase change } \\
& -\left\langle\rho_{0}^{s}\left(\vec{u}_{0}-\vec{u}_{0}^{s}\right) \cdot \nabla X_{k}\right\rangle & & \text { mass exchange } \\
& +\dot{R}_{k}^{s} & & \text { chemical reactions }
\end{aligned}
$$


where we have defined the average generation rate in phase $k$ due to chemical reactions as $\dot{R}_{k}^{s} \equiv \frac{\left\langle X_{k} \dot{r}^{s}\right\rangle}{\alpha_{k}}$.

\section{Momentum Balance}

The averaged momentum balance equation results from the generic, averaged balance equation (20) with the assignment of

$$
\psi_{0}=\vec{u}_{0} \quad J_{0}=\sim_{\sim} \quad g_{0}=\vec{g}_{0}
$$

to give

$$
\frac{\partial \alpha_{k} \rho_{k} \vec{u}_{k}}{\partial t}+\nabla \cdot \alpha_{k} \rho_{k} \vec{u}_{k} \vec{u}_{k}=\nabla \cdot \alpha_{k}\left(\underset{\sim k}{T_{k}}+T_{\sim}^{\text {Fluct }}\right)+\alpha_{k} \rho_{k} \vec{g}_{k}+\Omega_{k}^{\text {mom }}+\vec{u}_{k}^{\text {int }} \Omega_{k}^{\text {mass }}
$$

where the fluctuating stress $\underset{\sim}{T}$ Fluct and the interfacial momentum source $\Omega_{k}^{\text {mom }}$ are given by

$$
{\underset{\sim}{T}}_{\sim}^{\text {Fluct }} \equiv-\frac{\left\langle X_{k} \rho_{0} \vec{u}_{k}^{\prime} \vec{u}_{k}^{\prime}\right\rangle}{\alpha_{k}} \quad \Omega_{k}^{m o m} \equiv-\left\langle\underset{\sim 0}{T} \cdot \nabla X_{k}\right\rangle .
$$

The averaged interfacial momentum balance constraint (jump condition) is

$$
\sum_{k=1}^{\text {no. of phases }} \Omega_{k}^{\text {mom }}+\vec{u}_{k}^{\text {int }} \Omega_{k}^{\text {mass }}=\vec{M}_{\text {surfacetension }},
$$

where $\vec{M}_{\text {surface tension }}$ is the interfacial momentum source, i.e. surface tension source.

\section{Energy Conservation}

The assignment of

$$
\psi_{0}=E_{0}=e_{0}+\frac{1}{2} \vec{u}_{0} \cdot \vec{u}_{0} \quad J_{0}=T_{\sim} \cdot \vec{u}_{0}+\vec{q}_{0} \quad g_{0}=\vec{g}_{0} \cdot \vec{u}_{0}+\varepsilon_{0}
$$

to the variables of the generic, averaged balance equation (20) gives the averaged energy conservation equation 


$$
\begin{aligned}
\frac{\partial}{\partial t} \alpha_{k} \rho_{k}\left(e_{k}+\frac{1}{2} \vec{u}_{k} \cdot \vec{u}_{k}+e_{k}^{F l u c t}\right)+\nabla \cdot \alpha_{k} \rho_{k} \vec{u}_{k}\left(e_{k}+\frac{1}{2} \vec{u}_{k} \cdot \vec{u}_{k}+e_{k}^{\text {Fluct }}\right)= & \nabla \cdot\left[\alpha_{k}\left({\underset{\sim}{k}}_{k}+T_{\sim}^{\text {Fluct }}\right) \cdot \vec{u}_{k}\right] \\
& -\nabla \cdot \alpha_{k}\left(\vec{q}_{k}+\vec{q}_{k}^{\text {Fluct }}\right)+\alpha_{k} \rho_{k}\left(\varepsilon_{k}+\vec{g}_{k} \cdot \vec{u}_{k}\right) \\
& +\Omega_{k}^{\text {energy }}+\Omega_{k}^{\text {mom }} \cdot \vec{u}_{k}^{\text {int }} \\
& +\Omega_{k}^{\text {mass }}\left(e_{k}^{\text {int }}+\frac{1}{2} \vec{u}_{k}^{\text {int }} \cdot \vec{u}_{k}^{\text {int }}\right)
\end{aligned}
$$

where

$$
\begin{aligned}
& e_{k}^{\text {Fluct }} \equiv \frac{1}{2} \frac{\left\langle X_{k} \rho_{0} \vec{u}_{k}^{\prime} \cdot \vec{u}_{k}^{\prime}\right\rangle}{\alpha_{k} \rho_{k}} \quad \text { fluctuation kinetic energy } \\
& \vec{q}_{k}^{\text {Fluct }} \equiv \underbrace{\frac{\left\langle X_{k} \rho_{0} \vec{u}_{k}^{\prime} e_{k}^{\prime}\right\rangle}{\alpha_{k}}}_{\text {fluctution internal energy flux }}+\underbrace{\frac{\left\langle X_{k} T_{0} \cdot \vec{u}_{k}^{\prime}\right\rangle}{\alpha_{k}}}_{\text {fluctuation shear working }}+\underbrace{\frac{1}{2} \frac{\left\langle X_{k} \rho_{0} \vec{u}_{k}^{\prime}\left(\vec{u}_{k}^{\prime} \cdot \vec{u}_{k}^{\prime}\right)\right\rangle}{\alpha_{k}}}_{\text {fluctuation kinetic energy flux }} \text { fluctuation energy flux } \\
& \varepsilon_{k} \equiv \frac{\left\langle X_{k} \rho_{0} \varepsilon_{0}\right\rangle}{\alpha_{k} \rho_{k}} \quad \text { energy source } \\
& \Omega_{k}^{\text {energy }} \equiv\left\langle\vec{q}_{0} \cdot \nabla X_{k}\right\rangle \quad \text { interfacial heat source } \\
& \Omega_{k}^{m o m} \cdot \vec{u}_{k}^{i n t} \equiv-\left\langle\underset{\sim 0}{T} \cdot \vec{u}_{0} \cdot \nabla X_{k}\right\rangle \quad \text { interfacial work } .
\end{aligned}
$$

The averaged interfacial energy balance constraint (jump condition) is

$$
\sum_{k=1}^{\text {no. of phases }} \Omega_{k}^{\text {energy }}+\Omega_{k}^{\text {mom }} \cdot \vec{u}_{k}^{\text {int }}+\Omega_{k}^{\text {mass }}\left(e_{k}^{\text {int }}+\frac{1}{2} \vec{u}_{k}^{\text {int }} \cdot \vec{u}_{k}^{\text {int }}\right)=\xi \text {, }
$$

where $\xi$ is the interfacial energy source.

\section{Entropy Inequality}

The local form of the entropy inequality (8), sometimes called the "Second Law of Thermodynamics," is used to place restrictions on the constitutive relations used to give unique phase or material behaviors. With the assignment of 


$$
\psi_{0}=s_{0} \quad J_{0}=-\frac{\vec{q}_{0}}{\theta_{0}} \quad g_{0}=\frac{\varepsilon_{0}}{\theta_{0}}
$$

to the variables of the generic, averaged balance relationship (20) the averaged entropy inequality results,

$$
\frac{\partial \alpha_{k} \rho_{k} s_{k}}{\partial t}+\nabla \cdot \alpha_{k} \rho_{k} s_{k} \vec{u}_{k} \geq \nabla \cdot \alpha_{k}\left(\Phi_{k}+\Phi_{k}^{\text {Fluct }}\right)+\alpha_{k} \rho_{k} S_{k}+\Omega_{k}^{\text {entropy }}+\Omega_{k}^{\text {mass }} s_{k}^{\text {int }}
$$

where

$$
\begin{gathered}
\vec{\Phi}_{k} \equiv-\frac{\left\langle X_{k} \frac{\vec{q}_{0}}{\theta_{0}}\right\rangle}{\alpha_{k}} \quad \text { entropy flux } \\
\vec{\Phi}_{k}^{\text {Fluct }} \equiv-\frac{\left\langle X_{k} \rho_{0} s_{k}^{\prime} \vec{u}_{k}^{\prime}\right\rangle}{\alpha_{k}} \quad \text { fluctuation entropy flux } \\
S_{k} \equiv \frac{\left\langle X_{k} \frac{\rho_{0} \varepsilon_{0}}{\theta_{0}}\right\rangle}{\alpha_{k} \rho_{k}} \quad \text { entropy source } \\
\Omega_{k}^{\text {entropy }} \equiv\left\langle\frac{\vec{q}_{0}}{\theta_{0}} \cdot \nabla X_{k}\right\rangle \quad \text { interfacial entropy source. }
\end{gathered}
$$

This entropy inequality corresponds to what Drew and Passman (1999) call the microscopic entropy inequality. A macroscopic entropy inequality can be obtained by summing inequalities (30) over all of the phases or materials present in the mixture [Truesdell 1984 and the other authors contained therein]. The macroscopic entropy inequality is useful for placing restrictions on the phasic or material interaction constitutive relations. The averaged interfacial entropy inequality (jump condition) is

$$
\sum_{k=1}^{\text {no.of phases }} \Omega_{k}^{\text {entropy }}+\Omega_{k}^{\text {mass }} s_{k}^{\text {int }} \geq 0 .
$$




\section{Acknowledgements}

The author would like to acknowledge the U.S. Department of Energy, Office of Environmental Management for majority funding of this research as part of the Environmental Systems Research Program during Fiscal-Year 2001. The author also acknowledges Bechtel National Incorporated for funding a portion of the writing as part of the Corporate Funded Research and Development Program during Fiscal-Year 2002. This research was conducted under DOE Idaho Operations Office Contract DE-AC07-99ID13727.

\section{References}

Dobran, F., Theory of Structured Multiphase Mixtures, Springer-Verlag, Berlin, 1991.

Drew, D.A., "Mathematical Modeling of Two-Phase Flow," Ann. Rev. Fluid Mech., V. 15, 1983.

Drew, D.A. and Lahey, R.T., "Analytical Modeling of Multiphase Flow," in Particulate TwoPhase Flow, ed. M.C. Roco, Butterworth-Heinemann, Boston, 1993.

Drew, D.A. and Passman, S.L., Theory of Multicomponent Fluids, Springer-Verlag, New York, 1999.

Gelfand, I.M. and Shilov, G.E., Generalized Functions, Vol. I, Academic, New York, 1964.

Ishii, M., Thermo-Fluid Dynamic Theory of Two-Phase Flow, Eyrolles, Paris, 1975.

Kashiwa, B.A. and Rauenzahn, R.M., "A Multimaterial Formalism," Numerical Methods in Multiphase Flows, ASME FED-Vol. 185, p.149-157, 1994.

Kataoka, I. and Serizawa, A., "Interfacial Area Concentration and Its Roles in Local Instant Formulation of Two-Phase Flow," in Transient Phenomena in Multiphase Flow, ed. N.H. Afgan, Hemisphere, New York, 1988.

Truesdell, C., Rational Thermodynamics, $2^{\text {nd }}$ edition, Springer-Verlag, New York, 1984.

Whitaker, S., The Method of Volume Averaging, Kluwer Academic, Dordrecht, 1999. 


\section{Appendix: Moment Evolution Equation}

It is critical that special attention be given to functional dependencies in deriving the moment evolution equation. Let us begin first by defining the ensemble average of some property $Q_{0}$ as

$$
\left\langle Q_{0}\right\rangle(\vec{x}, t) \equiv \int Q_{0}\left(\Gamma_{0}\right) f\left(\vec{x}, t, \Gamma_{0}\right) d \Gamma_{0},
$$

where, for example, $\Gamma_{0}=\left\{\rho_{0}, \vec{u}_{0}, e_{0}, X_{1}, X_{2}, \cdots\right\}$. Note that, while $Q_{0}$ depends explicitly upon the state $\Gamma_{0}$, its average value $\left\langle Q_{0}\right\rangle$ depends upon position $\vec{x}$ and time $t$, which it acquired from the probability function $f\left(\vec{x}, t, \Gamma_{0}\right)$. In fact, $\left\langle Q_{0}\right\rangle$ loses its explicit dependence upon $\Gamma_{0}$ by its very definition as an integral over all possible states $\Gamma_{0}$.

The variation of the probability is

$$
d f=\frac{\partial f}{\partial t} d t+\frac{\partial f}{\partial \vec{x}} \cdot d \vec{x}+\frac{\partial f}{\partial \Gamma_{0}} \cdot d \Gamma_{0},
$$

so the time variation of the probability can be written

$$
\frac{d f}{d t}=\frac{\partial f}{\partial t}+\vec{u}_{0} \cdot \nabla f+\dot{\Gamma}_{0} \cdot \frac{\partial f}{\partial \Gamma_{0}} .
$$

If we now multiply this equation by $Q_{0}\left(\Gamma_{0}\right)$ and integrate over $\Gamma_{0}$-space we get

$$
\int Q_{0} \frac{d f}{d t} d \Gamma_{0}=\int Q_{0} \frac{\partial f}{\partial t} d \Gamma_{0}+\int Q_{0} \vec{u}_{0} \cdot \nabla f d \Gamma_{0}+\int Q_{0} \Gamma_{0} \cdot \frac{\partial f}{\partial \Gamma_{0}} d \Gamma_{0}
$$

Let us now further examine each term of this expression. Since $Q_{0}$ does not depend explicitly on time, $t$,

$$
\begin{aligned}
\int Q_{0}\left(\Gamma_{0}\right) \frac{\partial f}{\partial t} d \Gamma_{0} & =\int \frac{\partial\left(Q_{0} f\right)}{\partial t} d \Gamma_{0} \\
& =\frac{\partial}{\partial t} \int Q_{0} f d \Gamma_{0} \\
& =\frac{\partial}{\partial t}\left\langle Q_{0}\right\rangle(\vec{x}, t) .
\end{aligned}
$$

Similarly, since $Q_{0}$ is independent of $\vec{x}$, we have 


$$
\begin{aligned}
\int Q_{0} \vec{u}_{0} \cdot \nabla f d \Gamma_{0} & =\int \nabla\left(Q_{0} f\right) \cdot \vec{u}_{0} d \Gamma_{0} \\
& =\int \nabla \cdot\left(Q_{0} \vec{u}_{0} f\right) d \Gamma_{0}-\int Q_{0} f\left(\nabla \cdot \vec{u}_{0}\right) d \Gamma_{0} \\
& =\nabla \cdot \int Q_{0} \vec{u}_{0} f d \Gamma_{0}-\int Q_{0}\left(\nabla \cdot \vec{u}_{0}\right) f d \Gamma_{0} \\
& =\nabla \cdot\left\langle Q_{0} \vec{u}_{0}\right\rangle(\vec{x}, t)-\left\langle Q_{0} \nabla \cdot \vec{u}_{0}\right\rangle(\vec{x}, t) .
\end{aligned}
$$

We also know that the $\dot{\Gamma}_{0}$ are independent of $\Gamma_{0}$, so

$$
\begin{aligned}
\int Q_{0} \dot{\Gamma}_{0} \cdot \frac{\partial f}{\partial \Gamma_{0}} d \Gamma_{0} & =\dot{\Gamma}_{0} \cdot \int Q_{0} \frac{\partial f}{\partial \Gamma_{0}} d \Gamma_{0} \\
& =\dot{\Gamma}_{0} \cdot\left[\int \frac{\partial\left(Q_{0} f\right)}{\partial \Gamma_{0}} d \Gamma_{0}-\int f \frac{\partial Q_{0}}{\partial \Gamma_{0}} d \Gamma_{0}\right] \\
& =\left.\dot{\Gamma}_{0} \cdot Q_{0} f\right|_{\text {limit of } \Gamma_{0}}-\int \dot{\Gamma}_{0} \cdot \frac{\partial Q_{0}}{\partial \Gamma_{0}} f d \Gamma_{0} \\
& =0-\left\langle\dot{\Gamma}_{0} \cdot \frac{\partial Q_{0}}{\partial \Gamma_{0}}\right\rangle \\
& =-\left\langle\frac{\partial Q_{0}}{\partial \Gamma_{0}} \cdot \frac{d \Gamma_{0}}{d t}\right\rangle \\
& =-\left\langle\frac{d Q_{0}}{d t}\right\rangle=-\left\langle\dot{Q}_{0}\right\rangle(\vec{x}, t) .
\end{aligned}
$$

As long as the $Q_{0}$ are physically conserved quantities we also have

$$
\int Q_{0} \frac{d f}{d t} d \Gamma_{0}=0
$$

Putting all these expressions back into the integral equation above gives the moment evolution equation (11)

$$
\frac{\partial\left\langle Q_{0}\right\rangle}{\partial t}+\nabla \cdot\left\langle Q_{0} \vec{u}_{0}\right\rangle=\left\langle\dot{Q}_{0}+Q_{0} \nabla \cdot \vec{u}_{0}\right\rangle .
$$

【| Notice: By comparison with Equation (1.1) of [Kashiwa and Rauenzahn 1994],

$$
\frac{\partial\left\langle Q_{0}\right\rangle}{\partial t}+\nabla \cdot\left\langle Q_{0} \vec{u}_{0}\right\rangle=\left\langle\dot{\Gamma}_{0} \cdot \frac{\partial Q_{0}}{\partial \Gamma_{0}}\right\rangle+\int Q_{0} \frac{D f}{D t} d \Gamma_{0},
$$


theirs should read

$$
\begin{aligned}
\frac{\partial\left\langle Q_{0}\right\rangle}{\partial t}+\nabla \cdot\left\langle Q_{0} \vec{u}_{0}\right\rangle & =\left\langle\dot{\Gamma}_{0} \cdot \frac{\partial Q_{0}}{\partial \Gamma_{0}}\right\rangle+\left\langle Q_{0} \nabla \cdot \vec{u}_{0}\right\rangle+\int Q_{0} \frac{D f}{D t} d \Gamma_{0} \\
& =\left\langle\dot{Q}_{0}+Q_{0} \nabla \cdot \vec{u}_{0}\right\rangle+\int Q_{0} \frac{D f}{D t} d \Gamma_{0}
\end{aligned}
$$

to be correct. \ 